\title{
DÍPTEROS CALIPTRADOS (MUSCIDAE E ANTHOMYIIDAE) DA REGIÃO METROPOLITANA DO RIO DE JANEIRO, RJ. II. ATRATIVIDADE E FREQÜÊNCIA SAZONAL' ${ }^{1}$
}

\author{
José Mario d'Almeida ${ }^{2}$
}

\begin{abstract}
DIPTERA, CALYPTRATAE (MUSCIDAE E ANTHOMYIIDAE) FROM THE METROPOLITAN AREA OF RIO DE JANEIRO, RJ. II. ATRACTIVITY AND SAZONAL FREQUENCY. Nine thousand, six hundred and ninety-one flies of the family Muscidae ( 47 species) and Anthomyiidae ( 2 species) were captured. Flies were collected in three ecologically distinct areas (urban, rural and forest) within the metropolitan region of the city of Rio de Janeiro using as baits decomposing fish, bovine liver, fresh human faeces and mashed banana with sugar. Fish attracted the highest number of flies all over the year. Large number of flies were always collected in the summer and spring but reduced in the winter. Among several species of flies, Atherigona orientalis Schiner, 1868, Musca domestica Linnaeus, 1758 and Synthesiomya nudiseta (Wulp, 1883) were always frequent.
\end{abstract}

KEY WORDS. Diptera, Muscidae, Anthomyiidae, atractivity, sazonality

Os dípteros muscóides são atraídos por diversas iscas, para a oviposição e/ou alimentação, como também pelos "sítios de cópula" que se formam nestes substratos (D'ALMEIDA, 1989). Com o presente trabalho, desenvolvido em três áreas ecológicas distintas do Rio de Janeiro, objetivou-se estudar a atratividade que diferentes iscas exercem sobre moscas das familias Muscidae e Anthomyiidae, assim como, a influência das estações do ano sobre a atratividade das mesmas.

\section{MATERIAL E MÉTODOS}

As coletas efetuadas, simultaneamente, em 3 áreas ecológicas distintas da região metropolitana do Rio de Janeiro (urbana, rural e florestal), foram realizadas durante 20 dias de cada estação do ano (Inverno: 20.7 a 10.8.81; Primavera: 20.10 a 10.11 .81 ; Verão: 10 a 17.1 e 1 a 13.2 .82 e Outono: 20.4 a 10.5.82). Em cada ambiente foram distribuídas oito armadilhas (modelo descrito por D'ALMEIDA \& LOPES, 1983), duas para cada tipo de isca: peixe em decomposição (sardinha), fígado bovino em decomposição, fezes humanas frescas e banana d'água amassada com rapadura. A troca das iscas e a coleta

1) Parte da Tese de Mestrado, Departamento de Parasitologia da Universidade Federal Rural do Rio de Janeiro, pesquisa desenvolvida no Museu Nacional.

2) Laboratório de Entomologia Médica, Instituto de Biofísica Carlos Chagas Filho, Universidade Federal do Rio de Janeiro, 21949-900 Rio de Janeiro, Rio de Janeiro, Brasil. 
das moscas eram realizadas a cada 48 horas.

\section{RESULTADOS E DISCUSSÃO}

Foram coletados 9.691 exemplares, pertencentes a 47 espécies de Muscidae (9.657 espécimens) e 2 de Anthomyiidae (34 espécimens). As espécies estão nominadas a seguir, constando entre parênteses a quantidade de exemplares de acordo com as iscas usadas $(\mathrm{P}=$ peixe, $\mathrm{Fg}=$ fígado, $\mathrm{Fz}=$ fezes, $\mathrm{B}=$ banana $\mathrm{e} \mathbf{T}=$ total).

Muscidae:

Atherigona orientalis Schiner, $1868(\mathrm{P}=4.015, \mathrm{Fg}=758, \mathrm{Fz}=336, \mathrm{~B}=47$, $\mathrm{T}=5.156)$;

Ophyra aenescens (Wiedemann, 1839) $(\mathrm{P}=1.197, \mathrm{Fg}=515, \mathrm{Fz}=44$, $\mathrm{T}=1.756)$

Musca domestica Linnaeus, $1758(\mathrm{P}=1.023, \mathrm{Fg}=535, \mathrm{Fz}=40, \mathrm{~B}=25$, $\mathrm{T}=1.623)$; $\mathrm{T}=371)$

Synthesiomyia nudiseta (Wulp, 1883) $(\mathrm{P}=274, \mathrm{Fg}=64, \mathrm{Fz}=27, \mathrm{~B}=6$,

Ophyra sp. $(\mathrm{P}=229, \mathrm{Fg}=59, \mathrm{Fz}=30, \mathrm{~B}=1, \mathrm{~T}=319)$;

Morellia maculipennis (Macquart, 1846) $(\mathrm{Fz}=104, \mathrm{~B}=1, \mathrm{~T}=105)$;

Neomuscina pictipennis (Bigot, 1878$)(\mathrm{P}=2, \mathrm{Fz}=8, \mathrm{~B}=66, \mathrm{~T}=76)$;

Neomuscina sp. $(\mathrm{P}=10, \mathrm{Fg}=11, \mathrm{Fz}=17, \mathrm{~B}=24, \mathrm{~T}=62)$;

Morellia sp. $(\mathrm{Fz}=25)$;

Neomuscina atincta Snyder, $1949(\mathrm{Fz}=12, \mathrm{~B}=12, \mathrm{~T}=24)$;

Morellia flavicornis (Macquart, 1848) $(\mathrm{P}=1, \mathrm{Fz}=16, \mathrm{~B}=2, \mathrm{~T}=19)$;

Morellia bipuncta (Wiedemann, 1830) $(\mathrm{P}=1, \mathrm{Fz}=16, \mathrm{~T}=17)$;

Neomuscina instabilis Snyder, $1949(\mathrm{P}=4, \mathrm{Fg}=6, \mathrm{~B}=1, \mathrm{~T}=11)$;

Morellia ochricornis (Wiedmann, 1830) $(\mathrm{B}=10)$;

Neomuscina currani Snyder, $1949(\mathrm{Fg}=7)$;

Neomuscina mediana Snyder, $1949(\mathrm{P}=2, \mathrm{~B}=4, \mathrm{~T}=6)$;

Cyr toneurina sp. $(\mathrm{B}=6)$;

Cyrtoneurina prima (Malloch, 1924) $(\mathrm{B}=6)$;

Philornis univittata (Dodge, 1968) $(\mathrm{Fg}=2, \mathrm{Fz}=2, \mathrm{~B}=2, \mathrm{~T}=6)$;

Myospila obsoleta (Shannon \& Del Ponte, 1926) $(\mathrm{Fz}=5)$;

Morellia meridensis (Macquart, 1846) $(\mathrm{Fz}=5)$;

Graphomyia sp. $(\mathrm{Fg}=5)$;

Cyrtoneurina multomaculata (Stein, 1904) $(\mathrm{P}=1, \mathrm{Fg}=1, \mathrm{Fz}=2, \mathrm{~T}=4)$;

Neomuscina neosimilis Snyder, $1949(\mathrm{Fg}=1, \mathrm{Fz}=2, \mathrm{~T}=3)$;

Cyrtoneurina mellina Stein, $1918(\mathrm{Fz}=2)$;

Cyrtoneurina mimica Snyder, $1954(\mathrm{P}=1, \mathrm{Fz}=1, \mathrm{~T}=2)$;

Morellia nigricosta Hough, $1900(\mathrm{~B}=2)$;

Neomuscina similata Snyder, 1949;

Neomuscina stabilis (Stein, 1911) $(\mathrm{B}=2)$; 
Neomuscina atincticosta Snyder, $1949(\mathrm{Fz}=2)$;

Pseudoptilolepis fluminensis Albuquerque, $1954(\mathrm{Fg}=1, \mathrm{Fz}=1, \mathrm{~T}=2)$;

Cyrtoneurina varicolor (Hough, 1900) $(\mathrm{Fz}=1)$;

Cyrtoneurina trita (Stein, 1911) $(\mathrm{Fg}=1)$;

Dasymorellia sp. $(\mathrm{Fz}=1)$;

Graphomyia analis (Macquart, 1851) $(\mathrm{P}=1)$;

Morellia humeralis (Stein, 1918) $(\mathrm{Fz}=1)$;

Neomuscina vitoriae Lopes, $1982(\mathrm{Fz}=1)$;

Neomuscina capalta Snyder, $1949(\mathrm{Fg}=1)$;

Neomuscina inflexa (Stein, 1918) $(\mathrm{Fg}=1)$;

Philornis glaucinis (Dodge \& Critben, 1968) $(\mathrm{B}=1)$;

Philornis picci (Macquart, 1854) $(\mathrm{B}=1)$;

Philornis deceptivus (Dodge \& Critben, 1968) $(\mathrm{B}=1)$;

Pseudoptilolepis sp. $(\mathrm{Fg}=1)$;

Pseudoptilolepis fulvapeda Snyder, 1949 ( $\mathrm{P}=1)$;

Smithomyia sp. $(\mathrm{B}=1)$;

Xenothoracochaeta sp. $(\mathrm{B}=1)$;

Xenomorellia holti Malloch, $1923(\mathrm{Fz}=1)$;

Anthomyiidae:

Phaonantho devia Albuquerque, $1957(\mathrm{Fz}=23, \mathrm{~B}=2, \mathrm{~T}=28)$; $\mathrm{T}=6)$.

Craspedochaeta punctipennis (Wiedemann, 1830) $(\mathrm{P}=1, \mathrm{Fz}=1, \mathrm{~B}=4$;

Na figura 1 observa-se que peixe foi a isca mais atrativa, com $69,79 \%$ das moscas coletadas, resultados semelhantes foram constatados para califorídeos e sarcofagídeos (D'ALMEIDA E LOPES, 1983 e D'ALMEIDA, 1984) respectivamente. Utilizou-se peixe com 24 horas de exposição, o que de acordo com KAMAI \& SUENAGA (in FERREIRA, 1978) é a fase mais atrativa. Segundo CARVALHO et al. (1984), em Curitiba, Paraná, a isca preferida foi cebola em decomposição. Em Campinas, São Paulo, o peixe também foi a melhor isca (LINHARES, 1979).

Na tabela I é apresentada a distribuição dos muscóides, mais freqüentes, de acordo com as iscas, observando-se que $A$. orientalis e $M$. domestica foram capturadas em todas as iscas sendo que a primeira, só não foi a mais freqüente em áreas florestadas e de biologia pouco conhecida (D'ALMEIDA, 1982).

A preferência das espécies mais abundantes, pelas substâncias utilizadas, é apresentada na tabela II.

$\mathrm{Na}$ figura 2 pode-se constatar que as moscas foram mais freqüentes no verão e primavera, resultados semelhantes foram observados em São Carlos, São Paulo, por OLIVEIRA (1986). WATERHOUSE (1947), na Austrália e JIRÓN \& CARTÍN (1981) na Costa Rica, responsabilizaram a chuva como fator climático mais importante na mortalidade de larvas e pupas em carcaças de animais. No presente trabalho, de um modo geral, a precipitação e temperatura elevadas não influiram na freqüencia dos muscóides (Figs 2,3 e 4). 


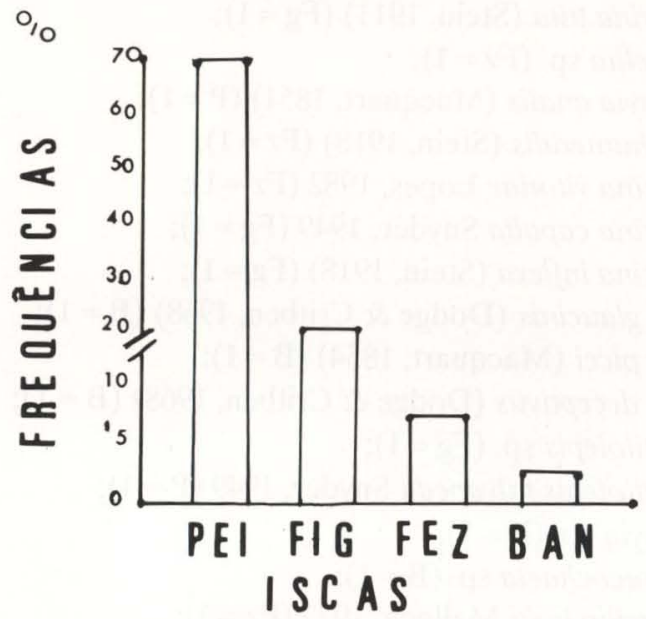

Fig. 1. Freqüência relativa de muscóides por iscas na região metropolitana do Rio de Janeiro, RJ ( $\mathrm{PEI}=$ peixe, $\mathrm{FIG}=$ fígado, $\mathrm{FEZ}=$ fezes, $\mathrm{BAN}=$ banana $)$.

Tabela I. Freqência relativa das principais espécies de muscídeos coletados em cada isca, na região metropolitana do Rio de Janeiro, $\mathrm{RJ}$ (50 ou mais exemplares).

\begin{tabular}{lcccc}
\hline \multicolumn{1}{c}{ ESPÉCIES } & PEIXE (\%) & FIGADO (\%) & FEZES (\%) & BANANA (\%) \\
\hline A. orientalis & 59,35 & 38,47 & 46,34 & 20,25 \\
M. domestica & 15,12 & 27,15 & 5,51 & 17,24 \\
O. aenescens & 17,69 & 26,14 & 6,06 & - \\
S. nudiseta & 4,05 & 3,24 & 3,72 & 2,58 \\
N. maculipennis & - & - & 14,34 & 0,43 \\
N. pictipennis & 0,02 & - & 1,10 & 28,44 \\
Outras & 3,74 & 4,88 & 22,89 & 30,67 \\
\hline
\end{tabular}

Na figura 3 é apresentada a freqüência sazonal das espécies mais freqüentes, onde se constata, que no verão e primavera as populações aumentam, com quedas acentuadas no inverno.

A. orientalis com $53,24 \%$ dos muscóides capturados, teve o peixe como isca mais atrativa (Tab. II), e com exceçẩo da banana, foi a espécie mais abundante nas iscas usadas (Tab. I). Segundo LINHARES (1979), A. orientalis e $M$. domestica foram as espécies mais freqüentes em Campinas, São Paulo, contribuindo com $91,70 \%$ dos muscóides estudados, e com abundância em carcaças de camundongos. De acordo com BOHART \& GRESSIT (1951) e POVOLNY (1971), suas larvas podem se alimentar de uma grande variedade de substâncias, desde carcaças de animais e vegetais em decomposição até fezes. 
D'ALMEIDA (1988) afirma que esta espécie cria-se com extrema facilidade em frutas em decomposição, observando-a em banana, mamão e tomate. Este muscídeo, no presente trabalho, foi mais freqüente no verão (Fig. 3) e em todas as estações do ano, o peixe foi a isca preferida (Tab. III); OLIVEIRA (1986) em um área rural de São Carlos, São Paulo, assinalou este muscídeo com mais freqüência na primavera.

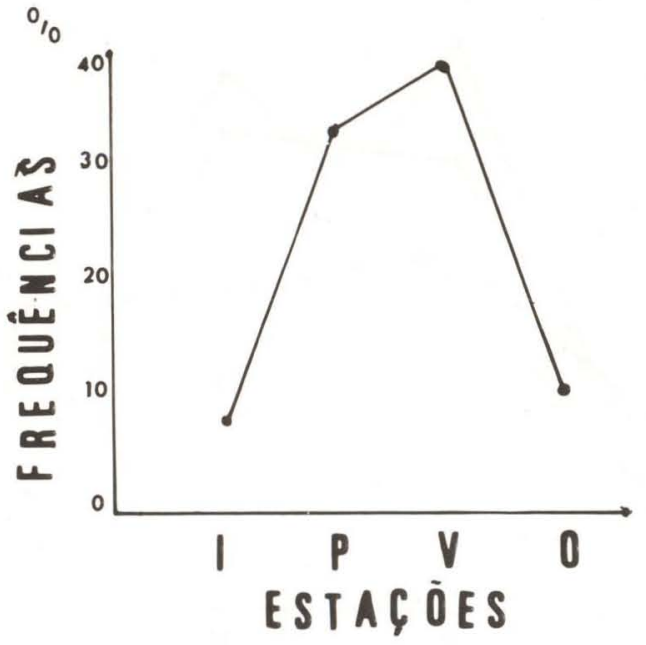

Fig. 2. Freqüência sazonal de muscóides capturados na região metropolitana do Rio de Janeiro, RJ ( $\mathrm{I}=$ inverno, $\mathrm{P}=$ primavera, $\mathrm{V}=$ verão, $\mathrm{O}=$ outono $)$.

Tabela II. Preferência das principais espécies de Muscidae pelos quatro tipos de iscas utilizadas, na região metropolitana do Rio de Janeiro (PEI = peixe, FIG = fígado, $\mathrm{FEZ}=$ fezes, $\mathrm{BAN}=$ banana). As iscas estão orientadas da esquerda para a direita em ordem decrescente de preferência.

\begin{tabular}{lllll}
\hline \multicolumn{1}{c}{ ESPÉCIES } & \multicolumn{4}{c}{ ISCAS } \\
\hline Atherigona orientalis & PEI & FIG & FEZ & BAN \\
Musca domestica & PEI & FIG & FEZ & BAN \\
Ophyra aenescens & PEI & FIG & FEZ & \\
Synthesiomya nudiseta & PEI & FIG & FEZ & BAN \\
\hline
\end{tabular}

Outra espécie abundante, que teve o peixe como isca mais atrativa, foi $O$. aenescens. Na tabela I, observa-se que foi ausente na banana e pouco freqüente nas fezes, o que diverge de LINHARES (1979), que afirmou ser ela freqüente em fezes. GREGOR (1975) constatou que este muscídeo prefere carnes, sendo fracamente atraído por fezes. CARVALHO et al. (1984) coletou apenas em peixe. BOHART \& GRESSIT (1951) chamam atenção para a grande variedade de substratos de criação utilizados por esta espécie, que vão de fezes 
humanas até vegetação em decomposição. D'ALMEIDA (1988), comparando uma série de substratos de criação, constatou que $O$. aenescens se desenvolve melhor em carnes em decomposição, prevalecendo em peixe. OLIVEIRA (1941) observou este muscóide criando-se em carcaças de animais marinhos depositados em areias úmidas de praias do Estado do Rio de Janeiro, ressaltando com o seu achado que essas larvas suportam bem a salinidade.

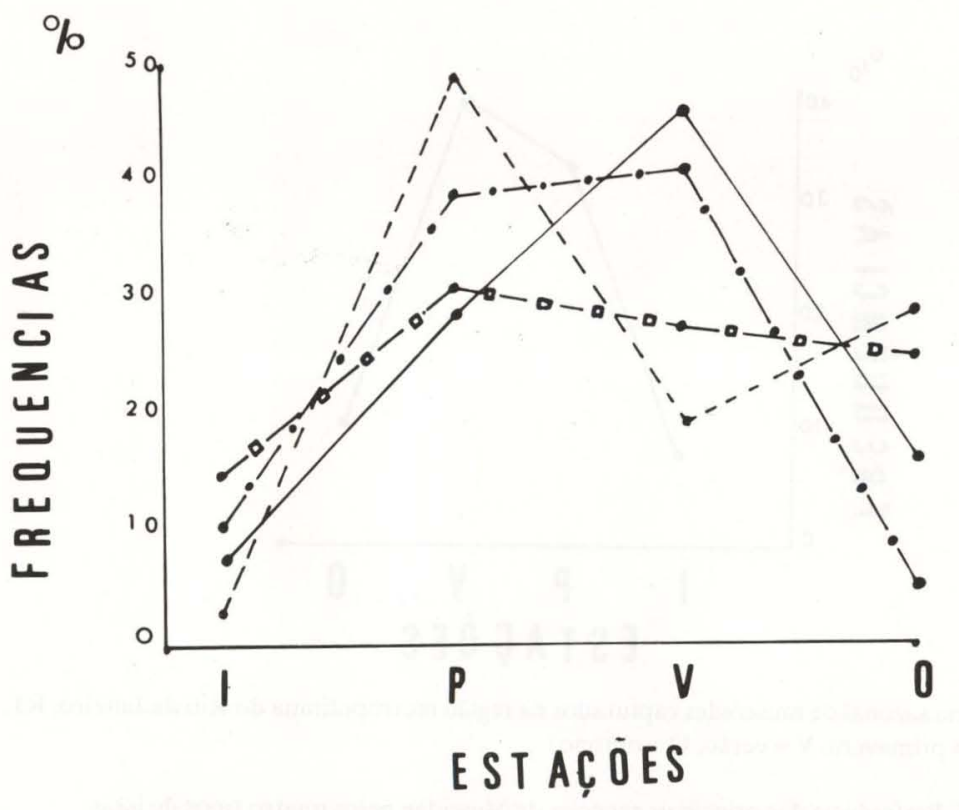
A. ORI ENT ALISI -$)$
D.AENESCENS (--)
M. DOMESTICA (- - )
S. NUDISEIA (-O-)

Fig. 3. Freqüência sazonal das espécies mais freqüentes de muscídeos capturados na região metropolitana do Rio de Janeiro, RJ.

O. aenescens foi mais freqüente na primavera (Fig. 3), com acentuado declínio no verão, o que pode estar relacionado com a alta pluviosidade nesta época (Fig. 4). Em todas as estações, o peixe foi a isca mais atrativa (Tab. III), LINHARES (1979) constatou resultados semelhantes sobre a variação sazonal.

No Rio de Janeiro, $M$. domestica foi mais freqüente em peixe, enquanto que em Campinas, São Paulo, foi abundante em vísceras de galinha e carcaça de camundongo. No presente trabalho, em Campinas (LINHARES, 1979) e em Curitiba, Paraná (CARVALHO et al., 1984), esta espécie prevaleceu no verão e primavera. Apenas no verão o fígado foi a isca mais atrativa (Tab. III), o que 
nos leva a sugerir que nesta estação, a temperatura e umidade mais elevadas, possam ter influído na atratividade deste substrato para $M$. domestica. Segundo POVOLNY (1971), esta mosca nos verões quentes dos trópicos e sub-trópicos, é rara no interior das residências, prevalecendo nos peridomicílios.

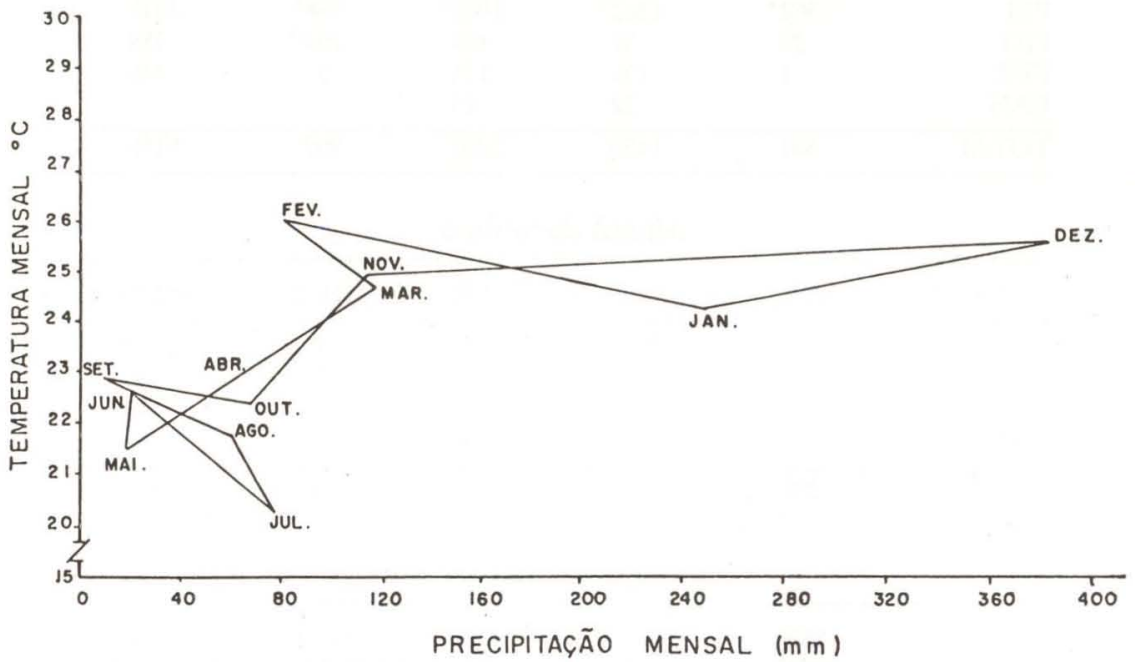

Fig. 4. Climatograma da cidade do Rio de Janeiro, RJ, no período de julho de 1991 a junho de 1992.

S. nudiseta foi outra espécie que teve o peixe como a isca mais atrativa (Tab. II), em todas as estações do ano (Tab. III). Segundo GREGOR (1975), em Cuba, este muscóide foi encontrado freqüentemente em carnes. Em Campinas, São Paulo, foi abundante em carcaças de camundongos (LINHARES, 1979).

Banana seguida de fezes humanas, foram as iscas mais atrativas para as moscas do gênero Morellia. Segundo LINHARES (1979), fezes foi a isca mais atrativa; banana não foi utilizada por este autor. Dentre as sete espécies do gênero capturadas no presente trabalho, apenas M. maculipennis, pelo número significativo de exemplares, merece destaque, coletada quase que exclusivamente em fezes. LOPES (1974), no Ceará, capturou este muscídeo com freqüencia em banana.

Outro gênero com grande número de espécies (doze), geralmente silvestres, foi Neomuscina, coletadas com abundância em banana, seguida pelas fezes. Dentre elas destaca-se $N$. pictipennis (76 exemplares), extremamente freqüente na banana $(86,84 \%)$.

Um interessante antomídeo silvestre foi Phaonantho devia, capturado exclusivamente nas fezes. LINHARES (1979), em Campinas, coletou este antomídeo em fezes e outras iscas, incidindo mais na primavera. 
Tabela III. Diferenças de atratividade das diversas iscas na coleta dos muscídeos mais freqüentes, nas quatro estações do ano $(\mathrm{PEI}=$ peixe, $\mathrm{FIG}=$ fígado, $\mathrm{FEZ}=$ fezes, $\mathrm{BAN}=$ banana, $\mathrm{INV}=$ inverno, $\mathrm{PRI}=$ primavera, $\mathrm{VER}=$ verão, $\mathrm{OUT}=$ outono).

\begin{tabular}{lrrrrr}
\multicolumn{7}{c}{ Atherigona orientalis } \\
\hline \multicolumn{1}{c}{ ISCAS } & INV & PRI & VER & OUT & TOTAL \\
\hline PEI & $362^{*}$ & $1262^{*}$ & $1802^{*}$ & $588^{*}$ & 4015 \\
FIG & 29 & 31 & 404 & 294 & 758 \\
FEZ & 1 & 135 & 175 & 27 & 336 \\
BAN & - & 22 & 25 & - & - \\
\hline TOTAL & 392 & 1450 & 2405 & 909 & 5156 \\
\hline
\end{tabular}

Musca domestica

\begin{tabular}{lccccc}
\hline \multicolumn{1}{c}{ ISCAS } & INV & PRI & VER & OUT & TOTAL \\
\hline PEI & $202^{*}$ & $599^{*}$ & $196^{*}$ & $26^{*}$ & 1023 \\
FIG & 2 & 3 & $476^{*}$ & $54^{*}$ & 535 \\
FEZ & - & 20 & 17 & 3 & 40 \\
BAN & - & 9 & 13 & 3 & 25 \\
\hline TOTAL & 204 & 631 & 702 & 86 & 1623 \\
\hline
\end{tabular}

Ophyra aenescens

\begin{tabular}{lrrrrr}
\hline \multicolumn{1}{c}{ ISCAS } & INV & PRI & VER & OUT & TOTAL \\
\hline PEI & 38 & $601^{*}$ & $231^{*}$ & $327^{*}$ & 1197 \\
FIG & 16 & 259 & 99 & 141 & 515 \\
FEZ & 2 & 15 & 8 & 19 & 44 \\
BAN & - & - & - & - & - \\
\hline TOTAL & 56 & 875 & 338 & 487 & 1756 \\
\hline
\end{tabular}

Synthesiomya nudiseta

\begin{tabular}{lccccc}
\hline \multicolumn{1}{c}{ ISCAS } & INV & PRI & VER & OUT & TOTAL \\
\hline PEI & $51^{*}$ & $107^{*}$ & $53^{*}$ & $63^{*}$ & 274 \\
FIG & - & - & $45^{*}$ & 19 & 64 \\
FEZ & 5 & 7 & 2 & 13 & 27 \\
BAN & - & 5 & 1 & - & 6 \\
\hline TOTAL & 56 & 119 & 101 & 95 & 371 \\
\hline
\end{tabular}

*Indicam iscas que apresentam maior atratividade, para cada espécie em cada estação do ano. Na análise foi feito teste de Chi-quadrado à nível de significância de $1 \%$.

Quanto às demais espécies, pelo pequeno número de exemplares, estão apenas nominadas na listagem citada.

Algumas considerações e sugestões podem ser enunciadas com relação à atratividade das iscas; certas substâncias que não são encontradas nas florestas e atraem moscas silvestres, possivelmente o fazem com vistas à alimentação, daí 
a necessidade de se analisar variados substratos encontrados naturalmente nas matas, assim como, desenvolver experimentos de laboratório que envolvam a capacidade das moscas de distinguir os substratos de criação das substâncias alimentares, como também o comportamento larvar frente a variados substratos.

AGRADECIMENTOS. O autor expressa os seus agradecimentos ao Prof. Hugo de Souza Lopes (in memorian) pela orientação da tese e por todos os ensinamentos proferidos, aos Profs. Rubens Pinto de Mello (FIOCRUZ) (correções e sugestões) e Reginaldo P. Brazil (Chefe do Laboratório de Entomologia Médica/UFRJ) pelas sugestões.

\section{REFERÊNCIAS BIBLIOGRÁFICAS}

BOHART, G.E. \& J.L. GRESSIT. 1951. Filth-inhabiting flies of Guam. Bull. Bernice P. Bishop. Museum, no. 204, VII + 152p.

CARVALHO, C.J.B. de; J.R. ALMEIDA \& C.B. de JESUS. 1984. Dípteros sinantrópicos de Curitiba e arredores (Paraná, Brasil). I. Muscidae. Rev. Brasil. Ent. 28 (4): 551-560.

D'ALMEIDA, J.M. 1982. Sinantropia em dípteros caliptratos na área metropolitana do Rio de Janeiro. Tese de Mestrado, não publicada, Universidade Federal Rural do Rio de Janeiro, Rio de Janeiro, RJ, 193p.

1984. Sinantropia de Sarcophagidae (Diptera) na região metropolitana do Rio de Janeiro. Arq. Univ. Fed. Rur. Rio de J. 7(2): 101-110.

1988. Substratos utilizados para a criação de dípteros caliptratos em uma área urbana do Município do Rio de Janeiro. Mem. Inst. Oswaldo Cruz 83: 201-206.

1989. Substratos utilizados para a criação de dípteros caliptratos no Jardim Zoológico do Rio de Janeiro (RIO-ZOO). Mem. Inst. Oswaldo Cruz 84(2): 257-264.

D'ALMEIDA, J.M. \& H.S. LOPES. 1983 Sinantropia de dípteros calitratos (Calliphoridae) no Estado do Rio de Janeiro. Arq. Univ. Fed. Rur. Rio de Janeiro 6: 39-48.

FERREIRA, M.J.M. 1978. Sinantropia de dípteros muscóides de Curitiba, Paraná. I. Calliphoridae. Rev. Brasil. Biol. 38 (2): 545-554.

GREGOR, F. 1975. Sinanthropy of Muscidae and Calliphoridae (Diptera) in Cuba. Folia Parasitol. , Praha, 22: 57-61.

JIRÓN, L.F. \& V.M. CARTÍN. 1981. Insect sucession in the decomposition of a mammal in Costa Rica. J. Entomol. Soc. N.Y. 89 (3): 158-165.

LINHARES, A.X. 1979. Sinantropia de dípteros muscóides de Campinas. Tese de mestrado, não publicada, Universidade Estadual de Campinas, Campinas, São Paulo, Brasil, 120p.

LOPES. H.S. 1974. Sarcophagidae flies (Diptera) from Pacatuba, State of Ceará, Brasil. Rev. Brasil. Biol. 34: 217-294.

OLIVEIRA, G.P. de. 1986. Distribuição sazonal de dípteros muscóides 
sinantrópicos, simbovinos e foréticos de Dermatobia hominis L.; em São Carlos, Estado de São Paulo. I. Estábulo. Arq. Biol. Tecnol. 29 (2): 311-325. OLIVEIRA, S.J. de. 1941. Sobre Ophyra aenescens (Wiedemann, 1830) (Diptera, Anthomyiidae). Arq. Zool. Est. São Paulo (Tomo XXV. Rev. Mus/Paulista) 2 (5): 341-356.

POVOLNY, D. 1971. Sinanthropy. p. 17-54. In: B. GREENBERG. Flies and Diseases. Vol. I: Ecology, classification and biotic association. Princetoun Univ. Press. Princeton, N.Y.

WATERHOUSE, D.F. 1947. The relative importance of live sheep and of carrion as breeding grounds for the Australian sheep blorufly Lucilia cuprina. Coun. Sci. Ind. Res. (Aust.) Bull. 217: 31 p.

Recebido em 29.V.1992; aceito em 10.I.1993. 